\title{
Intentional delay of surgery for acute type A dissection with stroke
}

Ikuo Fukuda, MD, a and Tomohiro Imazuru, MD, ${ }^{\text {b }}$ Amori and Tsukuba, Japan

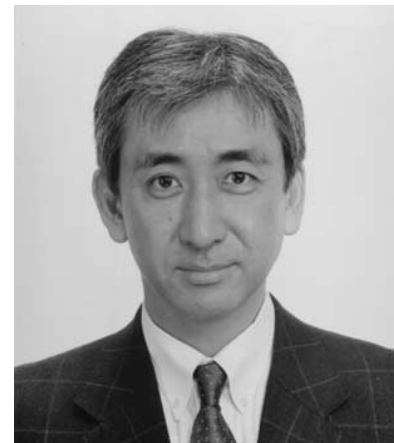

Dr Fukuda

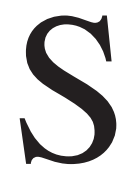

ince the brain is sensitive to ischemia-reperfusion injury, management of acute aortic dissection with cerebral infarction is controversial. ${ }^{1}$ We report on 4 patients with acute aortic dissection complicated by cerebral infarction.

\section{Clinical Summary}

The clinical course of 4 patients who had simultaneous onset of acute aortic dissection and stroke was reviewed (Table 1). The incidence of cerebral infarction was $8.2 \%$ among patients with Stanford type A dissection who were referred for surgery to Tsukuba Medical Center during the past 10 years. Although 1 patient died due to brain herniation, 3 patients successfully underwent reconstruction of the aortic arch in the chronic stage.

PATIENT 1. The patient was a 67-year-old man with coma and left hemiparesis due to acute type A aortic dissection. Computed tomography (CT) of the brain taken 10 hours after the onset revealed right cerebral infarction with swelling of the right hemisphere (Figure 1). Emergency surgery was postponed and an intentional delay strategy was employed. Since his condition deteriorated due to progressive brain edema, vigorous treatments for brain swelling including respiratory support were performed. The patient was weaned from the ventilator on the 18th day after onset. Although the patient's neurologic recovery was incomplete, reconstruction of the transverse arch was performed on the 83rd day. No neurologic deterioration was noted after surgery.

PATIENT 2. The patient was a 77-year-old woman with left hemiplegia. CT of the brain demonstrated diffuse right cerebral infarction with severe swelling of the right hemisphere. Urgent cerebral angiography revealed occlusion of the right common

From the First Department of Surgery, Hirosaki University School of Medicine, Aomori, Japan, ${ }^{\mathrm{a}}$ and Department of Cardiovascular Surgery, Tsukuba Medical Center Hospital, Tsukuba, Japan. ${ }^{\text {b }}$

Received for publication Aug 23, 2002; accepted for publication Sept 9, 2002.

Address for reprints: Ikuo Fukuda, MD, First Department of Surgery, Hirosaki University School of Medicine, 5 Zaifu-cho, Hirosaki, Aomori, 036-8562 Japan (E-mail: ikuofuku@cc.hirosaki-u.ac.jp).

J Thorac Cardiovasc Surg 2003;126:290-1

Copyright $(2) 2003$ by The American Association for Thoracic Surgery $0022-5223 / 2003 \$ 30.00+0$

doi:10.1016/S0022-5223(03)00358-1 carotid artery due to type A acute aortic dissection. Neurologic status of the patient deteriorated rapidly and she died on the second day due to brain hernia.

PATIENT 3. The patient was a 54-year-old man with left hemiplegia, motor weakness of the right upper extremity, and chest pain. Brain CT revealed cerebral infarction on the right parietal lobe with moderate local brain swelling. Aortography revealed dissection of the aortic arch, brachiocephalic artery, and left common carotid artery. Because motor weakness recovered almost completely, the patient successfully underwent reconstruction of the ascending aorta and the transverse aortic arch on the 41st day without any neurologic complication.

PATIENT 4. The patient was a 47-year-old woman with chest pain, loss of consciousness, and shock. Coronary angiography and aortography revealed dissection of the ascending aorta, transverse aortic arch, and patent coronary arteries. The brain CT revealed right cerebral infarction with swelling of the right hemisphere. Artificial ventilation was necessary for 2 weeks due to severe brain edema. On the 27th day, reconstruction of the ascending aorta and the transverse aortic arch was successfully completed without any deterioration of neurologic function.

\section{Discussion}

Involvement of arch branches in acute type A dissection has been reported with varying incidence, ranging from $5 \%$ to $46 \% .^{1,2}$ Surgical mortality and morbidity of patients with cerebral infarction in type A dissection was as high as $31 \%$ and $46 \%$, respectively. ${ }^{1}$ Reestablishment of flow into the infarcted area of the brain and use of high-dose heparin for extracorporeal circulation induces hemorrhagic infarcts and results in intractable brain edema. ${ }^{3}$ On the other hand, reversible ischemia due to temporal occlusion of the arch vessels may be observed in patients with acute aortic dissection. Therefore, differentiation between complete cerebral infarction and ischemia is vitally important. Even in irreversible ischemia, brain CT may not show any signs for several hours after occlusion of the artery. ${ }^{3}$ Piccione and colleagues ${ }^{4}$ reported the usefulness of intentionally delaying surgery for acute aortic dissection with stroke in a patient with Marfan syndrome. Deeb and associates $^{5}$ reported good results with a combination of early percutaneous reperfusion and delay of surgery until the reperfusion injury resolved. ${ }^{5}$

Fann and colleagues ${ }^{2}$ reported that 3 of 7 patients with acute aortic dissection complicated by stroke with persistent severe neurologic deficits died within 4 months (morbidity and mortality 

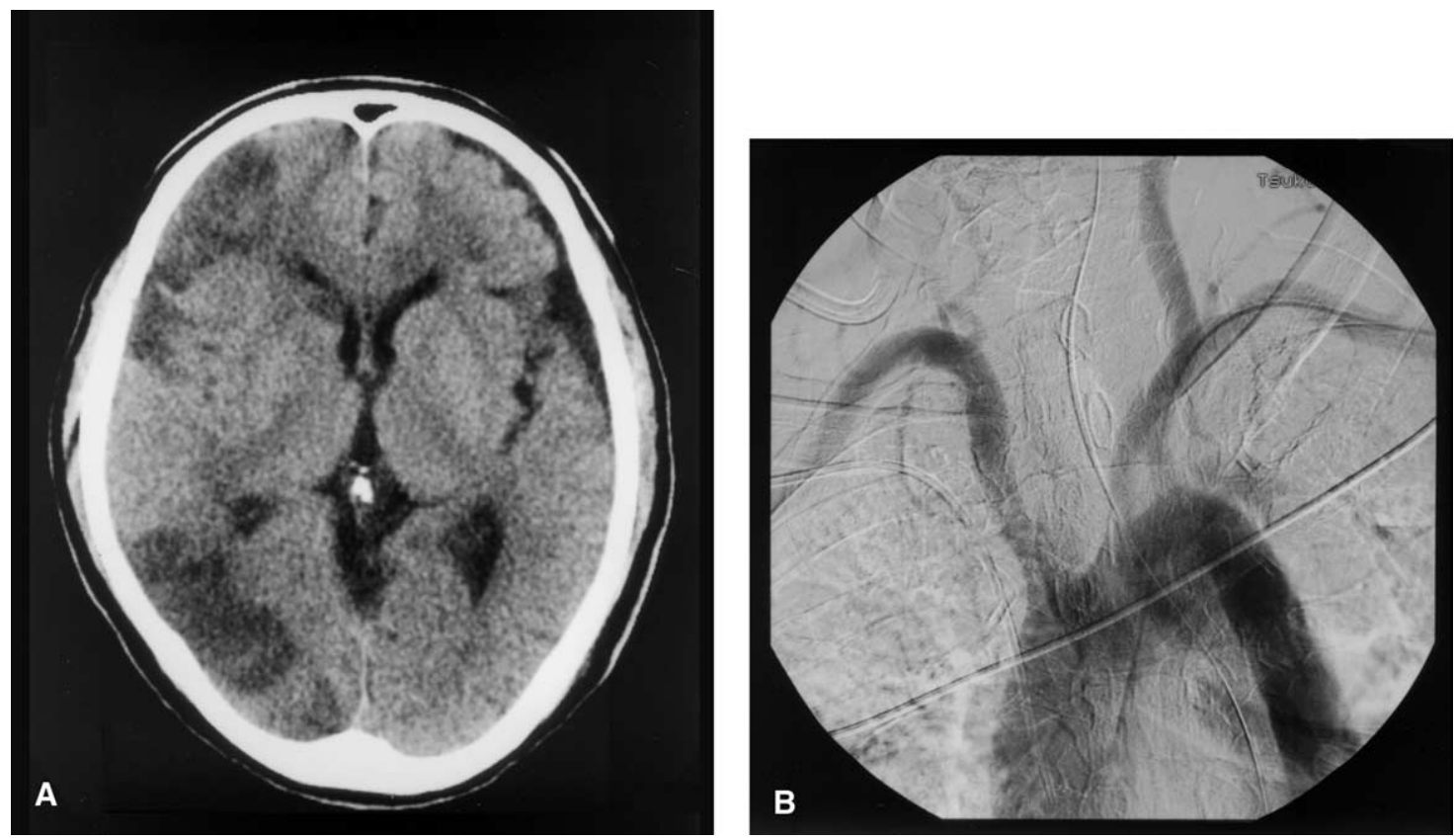

Figure 1. A, Brain CT of patient 1, revealing multiple low-density areas in the right hemisphere with moderate swelling. B, Aortogram of patient 1, revealing stenosis of the right common carotid artery due to dissection.

TABLE 1. Clinical presentation in 4 patients with complete cerebral infarction and acute aortic dissection

\begin{tabular}{|c|c|c|c|c|c|c|c|}
\hline $\begin{array}{l}\text { Patient } \\
\text { (age, sex) }\end{array}$ & $\begin{array}{l}\text { Tamponade } \\
\text { or shock }\end{array}$ & Initial tear & Site of cerebral infarction & $\begin{array}{l}\text { Brain } \\
\text { edema }\end{array}$ & $\begin{array}{c}\text { Onset-surgery } \\
\text { interval }\end{array}$ & Surgery & Outcome \\
\hline $1(67, M)$ & - & Descending aorta & Right temporoparietal lobe & Yes & $81 \mathrm{~d}$ & Total arch & Alive, 2 y Moderate disability \\
\hline $2(77, F)$ & - & Aortic arch & Right hemisphere & Yes & - & - & Died before surgery \\
\hline $3(54, M)$ & - & Aortic arch & Right parietal lobe & Yes & $41 \mathrm{~d}$ & Total arch & Alive, 6 y No disability \\
\hline $4(49, F)$ & + & Aortic arch & Right temporoparietal lobe & Yes & $27 \mathrm{~d}$ & Total arch & Alive, 4 y Moderate disability \\
\hline
\end{tabular}

Total arch, Total arch replacement.

$43 \%$ ). In this study, all patients had cerebral infarction with various degrees of brain edema. Although early reconstruction of the aorta may save the patient from rupture of the aorta, it may exacerbate brain edema and result in death. Appearance of a low-density area in brain CT means breakdown of brain tissue with surrounding edema. The interval between onset and restoration of cerebral blood flow takes several hours even if emergency surgery is conducted. Our patients had acceptable neurologic recovery and operative mortality even with extensive reconstruction of the transverse aortic arch.

In conclusion, intentional delay of surgery and observation with medical treatment is useful for patients who have acute aortic dissection with cerebral infarction.

\section{References}

1. Borst HG, Heinemann MK, Stone CD. Malperfusion of arch branches. In: Borst HG, editor. Surgical treatment of aortic dissection. New York: Churchill Livingstone; 1996. p. 251-4.

2. Fann J, Sarris G, Miller D, et al. Surgical management of acute aortic dissection complicated by stroke. Circl. 1989;80(Suppl I):I257-63.

3. Toole JF. Brain infarction: pathophysiology, clinical features, and management. In: Toole JF, Murros K, Biller J, editors. Cerebrovascular disorders. 5th ed. Philadelphia: Lippincott Williams \& Wilkins; 1999. p. 193-232.

4. Piccione W, Hamilton IN, Najafi H. Intentional delayed repair of acute dissection of the ascending aorta complicated by stroke. $J$ Thorac Cardiovasc Surg. 1995;109:807-8.

5. Deeb GM, Williams DM, Bolling SF, et al. Surgical delay for acute type A dissection with malperfusion. Ann Thorac Surg. 1997;64:1669-77. 